\title{
NUCLEAR ACTIVITY IN THE SEYFERT GALAXY NGC 1365
}

\author{
M. HJELM, P.O. LINDBLAD and S. JÖRSÄTER \\ Stockholm Observatory, 13396 Saltsjöbaden, Sweden
}
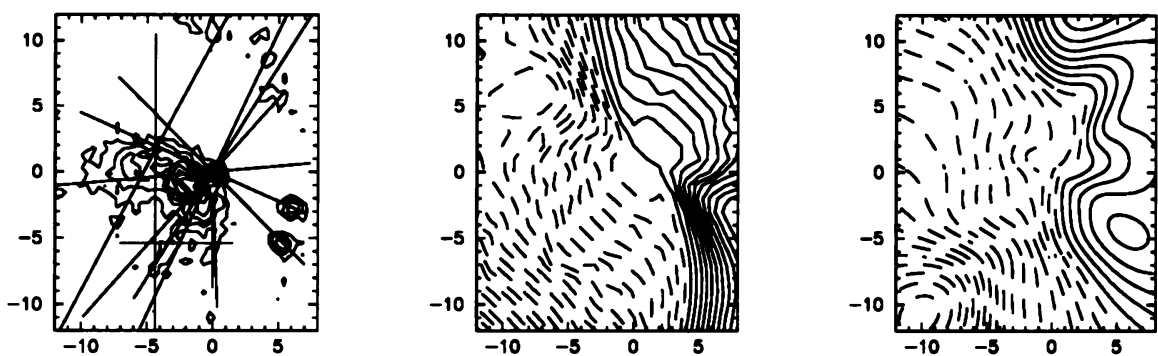

Fig. 1. a: Continuum-subtracted narrow band image in [OII] $\lambda 5007$. Superimposed are positions of spectra used in deriving the velocity fields. b: "Anomalous" velocity field. c: Model velocity field, in which the velocity, $v$, in the cone depends on the distance from the center, $r$, and on the angular distance, $t$, from the cone axis as where $t_{o}$ is half the opening angle of the cone. Density $=$ const $/ r^{4}$. The inclination of the cone axis to the line of sight is $40^{\circ}, t_{o}=45^{\circ}$ and the cones are directed $180^{\circ}$ away from each other. Units on the axes in all three plots are arcseconds offset from the center. In $b$ and $c$ the dashed lines indicate blueshifted velocities and the velocity interval is $10 \mathrm{kms}^{-1}$.

NGC 1365 is a barred Seyfert 1.5 galaxy. Fig 1 a is a contour plot of an [OIII] $\lambda 5007$ image (Jörsäter \& Lindblad 1989), showing a plume-like structure pointing towards $\mathrm{SE}$ in the direction of the minor axis of the galaxy, which also is the direction of the steepest velocity gradient in $[\mathrm{OIII}] \lambda 5007$. The plume is roughly aligned with a radio feature in the 6 and $20 \mathrm{~cm}$ continuum (see Sandqvist et al., this volume). The [OIII]-emission on the NW side is weaker. This plume-like structure is not seen in $\mathrm{H} \alpha$.

The velocity field of the inner parts of NGC 1365 can be divided into two separa te components. The low excitation lines (eg. the Balmer lines) show the galactic rotation. The higher excitation lines (eg. the [OIII]-lines) show "anomalous" velocities that are blueshifted relative to the systemic velocity of the galaxy to the SE of the nucleus and redshifted to the NW (Fig. 1b). We model these "anomalous" velocities as a bi-conical accelerated outflow, in which the density is given by the equation of continuity (steady state) for a chosen velocity law in the cones. Fig 1c shows the velocity field derived from one of our preliminary models. For details and further discussion see Hjelm et al. 1994.

\section{References}

Hjelm M., Lindblad P.O. and Jörsäter S., 1994, in prep.

Jörsäter S. and Lindblad P.O.,1989, ESO Workshop on Extra Nuclear Activity, p 39.

448

T. J.-L. Courvoisier and A. Blecha: Multi-Wavelength Continuum Emission of AGN, 448.

(C) 1994 IAU. Printed in the Netherlands. 